\title{
Carbocysteine: A New Way for Stretch Marks Treatment
}

\author{
Francesco Scarci, Federico Mailland \\ Scientific Department, Polichem S.A., Lugano, Switzerland \\ Email: fscarci@polichem.com
}

Received 10 September 2015; accepted 28 November 2015; published 1 December 2015

Copyright (C) 2015 by authors and Scientific Research Publishing Inc.

This work is licensed under the Creative Commons Attribution International License (CC BY). http://creativecommons.org/licenses/by/4.0/

(c) $\underset{\mathrm{EY}}{\mathrm{i}}$ Open Access

\begin{abstract}
Background/Purpose: Although stretch marks (or striae) do not represent a medical problem, they are considered as the cause of psychological distress for women of any age that need to be treated. There are many cosmetic products or procedures claiming to improve the appearance of striae, but most of them are able to affect only striae appearance rather than physical characteristics or, even worse, they are provided with untoward effects. Our aim was to find a more efficacious and safe alternative to the existing tools. Methods: A randomized, blind observer study was conducted on 33 women to test the efficacy and safety of P-3059, a new product containing carboxymethylcysteine, Vitamin E and sweet almond oil. P-3059 was applied twice daily for 8 weeks and it was tested intra-subject in a split-body design versus an untreated area (negative control), comparing two symmetric areas with striae selected on the body/legs of each subject. The main study endpoint was the visual evaluation of striae, by means of the validated POSAS scale (consisting of two parts, OSAS and PSAS), at the end of treatment in comparison with baseline. Results: The comparison of the individual parameters of OSAS, showed significantly improved mean values for striae thickness, relief and pliability at the end of treatment $(p<0.05)$. The PSAS evaluation showed a statistically significant benefit in the improvement in thickness of the striae towards that of normal skin $(p<0.044)$. Conclusion: The new product based on carboxymethylcysteine leads to an improvement in the appearance and in the physical characteristics of striae, reducing their thickness and improving relief and pliability, as well as subjects satisfaction.
\end{abstract}

\section{Keywords}

Striae Distensae, Stretch Marks, Emollient, Carbocysteine, Sweet Almond Oil

\section{Introduction}

Striae distensae or stretch marks represent a common skin condition that does not induce any significant medi-

How to cite this paper: Scarci, F. and Mailland, F. (2015) Carbocysteine: A New Way for Stretch Marks Treatment. Journal of Cosmetics, Dermatological Sciences and Applications, 5, 275-282. http://dx.doi.org/10.4236/jcdsa.2015.54033 
cal problem, but can cause psychological distress, due to adverse appearance and physical characteristics such as thickness and suppleness, and is undesirable to those affected [1]. Caused by pregnancy, rapid weight gain or loss, or a growth spurt in the early teens, the physiological cause is reportedly linked to hormonal changes and their consequences in the skin metabolism of the affected area [2]. The occurrence of striae affects the abdomen and the breasts of pregnant women of all races in 50\% to $90 \%$ of cases [3]; in adolescents, in people overweight or in patients with excessive adrenal cortical activity, it affects thighs, hips, lower back and buttocks. Striae appear as depressed, reddish or purple lines (or in the worst cases as lesions), which later tend to gradually become white, smooth and shiny, or even wrinkled [4]. This is due to the breaks in the connective tissue. Indeed, skin distension may lead to excessive mast cell degranulation with subsequent damage to collagen and elastin [5]. Due to their physiological nature, they are considered as an aesthetic problem that can be treated with various modalities. Topical tretinoin for example, improves the appearance of striae and the improvement may persist for almost a year after discontinuation of therapy [6]. However, tretinoin may be the cause of irritation, as it indirectly increases sun sensitivity and fragility of the skin [7]. Lasers represent another option in treating striae and have minimal side effects but a moderately beneficial effect [8] [9]. Minimal pain and post treatment hyperpigmentation were the main adverse events reported in some clinical trials with lasers [10]. Abdominoplasty is a cosmetic surgery procedure used to make the abdomen thinner and more firm but it carries certain risks that may be serious or life-threatening [11]. Keratolytic and exfoliants have also been reported as useful due to their peeling effects. These products include salicylic and glycolic acid [6]. Massage with a silicone gel over a period of 6 weeks significantly increased the content of collagen in stretch marks over placebo, and decreased pliability [12]. Most of the above products may cause irritation and efficacy remains controversial [13]. There are numerous other cosmetic products that claim to improve the appearance of striae but most of these are not validated and/or only affect striae appearance rather than physical characteristics such as striae thickness and suppleness.

Thus, there is still an important medical need for topical preparations, which can be effectively and safely used for both improving the appearance of and minimizing the adverse physical characteristics of stretch marks.

The aim of this study was to assess the aesthetic efficacy and the safety profile of a new cosmetic product (P3059), based on carboxymethylcysteine (a very safe compound commonly used in the treatment of a long-term respiratory diseases such as chronic obstructive pulmonary disease [COPD]), Vitamin E and sweet almond oil, on reducing striae on the body and legs of female subjects over a period of 8 weeks of treatment. P-3059 has the capability to decrease the rigidity of protein structures, by breaking the disulphuric bonds, resulting in an increase in the extensibility of the protein molecules.

\section{Materials and Methods}

A randomized, open-label, blind observer, intra-individual comparison study was performed, complying with the Declaration of Helsinki and principles of GCP, at one private clinic in Schenefeld/Hamburg in Winter 2014. The Freiburg Ethics Commission International approved the study. The subjects gave their consent to the study in writing, before entering the trial.

The study had to recruit 33 Caucasian female subjects from 18 to 65 years of age, with skin type I to III according to Fitzpatrick scale (used to classify the human skin color) and having two comparable areas with striae on the body/legs according to the Mallol score (Score 1 to 3, with 1 indicating the presence of few and thin striae and 3 the presence of many thick striae), due to either post pregnancy, weight training, weight gain or adolescent growth spurt. The main exclusion criteria included excessive loss of weight or weight gain within the last 2 months prior to and throughout the course of the study (less than $3 \mathrm{~kg}$ weight changes were allowed) and the presence of moles, tattoos, irritated skin, hairs etc. at the test area that could influence the investigation.

The subjects were instructed to avoid any exposure of the test area to artificial or natural UV light within the last 14 days prior to study start and for the whole study period. Moreover, they were instructed to discontinue the application of leave-on cosmetics (e.g. creams, lotions, sunscreens) in the test area within the last 5 days prior to study start and for the whole study period.

A sufficient volume of P-3059 had to be applied twice daily for 8 weeks by the subjects at home to adequately cover the whole test area (in the morning and in the evening), by means of a massage with the fingers for 5 minutes into the test area using a light pressure.

P-3059 was tested in a split-body design versus an untreated area (as negative control), choosing two compa- 
rable areas with striae to be assessed, selected on the body/legs of each subject (or one large area that could be appropriately allocated to test and control areas) by a trained clinical technician during the scheduled visit on Day 1 . The test areas had to be at least $5 \times 5 \mathrm{~cm}$ in size.

\subsection{POSAS Scale}

Subjects underwent an 8-week treatment, coming to the study site for the screening procedures (Day 1), after 4 weeks of treatment for a preliminary evaluation of treatment compliance and at the end of treatment for the visual evaluation of striae, performed according to the POSAS scale, in comparison with the baseline evaluation. The POSAS scale consists of two parts: the Observer Scar Assessment Scale (OSAS) and the Patient Scar Assessment Scale (PSAS). Both scales contain six items that are scored numerically on a ten-step scale with 10 indicating the worst imaginable striae or sensation [13]. Together they make up the "Total Score" of the Patient and Observer Scale; the total score for each of the scales consists of adding the scores of the six items (range 6 to 60 ). The lowest score, 6 , reflected normal skin, whereas the highest score, 60 , reflected the worst imaginable striae.

The objective assessment according to OSAS was performed by a Trained Evaluator, rating striae with regard to the following: vascularity, pigmentation, pliability, thickness, relief, surface area.

For each item of the OSAS, the following scale was used: from $1=$ Normal skin to $10=$ Worst striae imaginable.

To distinguish between the evaluation of vascularity (presence of vessels in striae tissue assessed by amount of redness) and pigmentation (brownish coloration of striae), a 3 mm-thick Plexiglas tool was used. The Plexiglas was pressed on the concerned areas assessing pigmentation of the striae and the surrounding skin. Then, vascularity was assessed by releasing the tool and looking at the capillary refill. For thickness, the average distance between the subcutical-dermal border and the epidermal surface of striae was considered. For the relief, the presence of surface irregularities was evaluated; for pliability the elasticity of striae was tested by wrinkling the striae between the thumb and index finger. Finally, the surface area of striae was evaluated in relation to the original affected area.

For the PSAS, the subjects were required to answer the following six questions:

a) Pain: are the striae painful?

b) Itching: are the striae itching?

c) Color (pigmentation and vascularity): is the color of the striae different from the color of your normal skin?

d) Pliability: is the stiffness of the striae different from your normal skin?

e) Thickness: is the thickness of the striae different from your normal skin?

f) Relief: are the striae more irregular than your normal skin?

The following scale was used: from $1=$ No, not at all (for a and b)/No, as normal skin (from c to f) to $10=$ Yes, very much (for a and b)/Yes, very different (from c to f).

\subsection{Statistical Analysis}

Treatments were randomly assigned to the left and right test area. The randomization was balanced 1:1, with the block size of 8 and was given according to a random vector with uniform distribution for a consecutive numbering of the subjects in this study.

Raw data for single items of OSAS and POSAS were listed by treatment and assessment time for Per Protocol (PP) population only. Separately, total scores for OSAS and POSAS, as well as differences to baseline for total scores, were calculated and listed by treatment and assessment time. Descriptive statistics (n, mean, standard deviation, standard error of the mean, median, minimum, maximum and $95 \%$ confidence limits) were given for raw data and calculated values. The mean values and differences from baseline were presented in bar charts by treatment and assessment time with 95\%-confidence intervals for total scores of OSAS and POSAS separately. A significance level of 0.05 (alpha) was chosen for statistical analysis. The comparison of treatments was performed on differences from baseline separately for total scores of OSAS and PSAS using paired t-Test. Further comparison between times within each treatment group was undertaken for total scores of OSAS and PSAS with paired t-Test. Differences between times were tested by Wilcoxon test for paired samples for the individual items of OSAS and PSAS, respectively. The proportions of subjects with improvement/worsening was processed between treatments by Fisher's Exact test. 


\section{Results}

Thirty-three subjects, with an average age of $33.1 \pm 7.4$ years (min age: 19; max age: 45; SEM: 1.29), were enrolled. One subject withdrew from the study and was excluded from PP population, being only included in the Safety population (SP).

The results are shown in Table 1.

For OSAS, the comparison of assessment times showed a significantly lower mean score for P-3059 on Day 57 compared to baseline (Day 1) (Different between times $-1.8, \mathrm{p}=0.009$ ). No real changes were found for the untreated area on Day 57 compared to Day 1 for OSAS (Different between times +0.3 , N.S.). The comparison between treatments showed a significantly higher decrease in favor of P-3059 ( $p<0.001)$.

For PSAS, a slight but not significant decrease of the mean score (Different between times - 1.3) was found for the untreated area on Day 57 compared to Day 1. A clearer decrease of the mean score was seen for P-3059 on Day 57 (Different between times - 2.2) compared to Day 1, but the level of significance was not achieved. No significant differences between treatments were found regarding PSAS.

The comparison of assessment times for the individual parameters of OSAS, showed significantly improved mean values for striae thickness, relief and pliability on Day 57 in comparison to Day 1 for P-3059 (Table 2).

Table 1. OSAS and PSAS-mean values \pm SD and comparison between times within treatment as well as between treatments by Paired $\mathrm{t}-\mathrm{Test}(\mathrm{N}=32)$.

\begin{tabular}{ccccccc}
\hline \multirow{2}{*}{ Parameter } & \multicolumn{2}{c}{ Mean values } & \multicolumn{3}{c}{ p-values } \\
\cline { 3 - 6 } & Time & \multicolumn{2}{c}{ P-3059 } & $\begin{array}{c}\text { Between } \\
\text { treatments }\end{array}$ & Untreated & P-3059 \\
\cline { 3 - 6 } & & Untreated & Peen times \\
\hline \multirow{2}{*}{ OSAS } & Day 1 & $23.3 \pm 6.5$ & $23.8 \pm 6.4$ & - & 0.524 & 0.009 \\
& Day 57 & $23.6 \pm 6.5$ & $22.0 \pm 6.6$ & $<0.001$ & & \\
\multirow{2}{*}{ PSAS } & Day 1 & $22.8 \pm 5.7$ & $22.8 \pm 5.6$ & - & 0.270 & 0.083 \\
& Day 57 & $21.5 \pm 6.3$ & $20.6 \pm 6.6$ & 0.207 & & \\
\hline
\end{tabular}

OSAS and PSAS Score: $6=$ normal skin to $60=$ worst striae imaginable.

Table 2. Parameters of OSAS-mean values and results for the comparison of treatments and measurement time points by Wilcoxon signed-ranks test $(\mathrm{N}=32)$.

\begin{tabular}{|c|c|c|c|c|c|c|}
\hline \multirow{3}{*}{$\begin{array}{c}\text { Parameter of } \\
\text { OSAS }\end{array}$} & \multirow{3}{*}{ Time } & \multicolumn{2}{|c|}{ Mean values } & \multicolumn{3}{|c|}{ p-values } \\
\hline & & \multirow[b]{2}{*}{ Untreated } & \multirow[b]{2}{*}{ P-3059 } & \multirow[b]{2}{*}{$\begin{array}{c}\text { Between } \\
\text { treatments }\end{array}$} & \multicolumn{2}{|c|}{ Between times } \\
\hline & & & & & Untreated & P-3059 \\
\hline \multirow{2}{*}{ Vascularity } & Day 1 & $2.9 \pm 1.0$ & $3.0 \pm 1.0$ & -- & \multirow{2}{*}{1.000} & \multirow{2}{*}{0.750} \\
\hline & Day 57 & $2.9 \pm 1.0$ & $3.0 \pm 1.0$ & 0.688 & & \\
\hline \multirow{2}{*}{ Pigmentation } & Day 1 & $2.8 \pm 1.0$ & $3.1 \pm 1.1$ & -- & \multirow{2}{*}{0.625} & \multirow{2}{*}{0.531} \\
\hline & Day 57 & $2.9 \pm 1.0$ & $3.0 \pm 1.0$ & 0.234 & & \\
\hline \multirow{2}{*}{ Thickness } & Day 1 & $4.2 \pm 1.3$ & $4.1 \pm 1.3$ & -- & \multirow{2}{*}{1.000} & \multirow{2}{*}{0.003} \\
\hline & Day 57 & $4.2 \pm 1.3$ & $3.6 \pm 1.2$ & 0.003 & & \\
\hline \multirow{2}{*}{ Relief } & Day 1 & $4.1 \pm 1.5$ & $4.2 \pm 1.5$ & -- & \multirow{2}{*}{1.000} & \multirow{2}{*}{0.034} \\
\hline & Day 57 & $4.2 \pm 1.5$ & $3.8 \pm 1.5$ & 0.038 & & \\
\hline \multirow{2}{*}{ Pliability } & Day 1 & $5.0 \pm 1.5$ & $5.2 \pm 1.6$ & -- & \multirow{2}{*}{1.000} & \multirow{2}{*}{$<0.001$} \\
\hline & Day 57 & $5.0 \pm 1.5$ & $4.4 \pm 1.6$ & $<0.001$ & & \\
\hline \multirow{2}{*}{ Surface Area } & Day 1 & $4.3 \pm 1.4$ & $4.3 \pm 1.3$ & -- & \multirow{2}{*}{0.109} & \multirow{2}{*}{0.973} \\
\hline & Day 57 & $4.5 \pm 1.4$ & $4.3 \pm 1.5$ & 0.129 & & \\
\hline
\end{tabular}

Score: 1 = normal skin to $10=$ worst striae imaginable. 
The differences on the three parameters were significant between treatments, too. Nofurther significant differences were observed.

In Figure 1, the counts of subjects with improvement and worsening regarding the OSAS parameters separately by treatment are shown including percentages.

In 53\% of the subjects, an improvement in skin thickness was found, an improvement in relief appeared in $44 \%$ of the subjects and an improvement in pliability was reported in 63\% of the subjects treated with P-3059. In the untreated area, a negligible proportion of subject with improvement was seen for the same parameters. Fisher's Exact test between treatment groups was: $\mathrm{p}=0.0007$ (thickness), $\mathrm{p}=0.0076$ (relief), $\mathrm{p}=0.0435$ (pliability).

PSAS, as for OSAS, is a composite endpoint. Table 3 presents the mean values for the scores of all PSAS parameters separately by treatment and measurement time points, the mean differences compared to baseline, and the results for the comparison of treatments and time points by Wilcoxon signed-ranks test.

The comparison of assessment times showed a significantly lower mean value in P-3059 group for the question "Is the thickness of the striae different from your normal skin" on Day 57 compared to baseline ( $p<0.044)$, indicating an improvement in thickness of the striae towards that of normal skin. Furthermore, a significantly lower mean value was determined for the question "Are the striae more irregular than your normal skin" for the untreated area on Day 57 compared to baseline, indicating a worsening in irregularity of the striae. The corresponding mean value for P-3059 just missed the level of significance and no further significant differences were found for the comparison of assessment times. The comparison of treatments showed no significant differences for the parameters of PSAS. In Figure 2, the counts of subjects with improvement and worsening regarding the PSAS parameters separately by treatment are shown, including percentages. No significant differences between treatments were detected by Fisher's test.

Half subjects or more than half-reported improvement in striae stiffness (50\%), improvement in striae thickness (56\%) and improvement in striae irregularity (66\%) for P-3059. For the untreated area, the improvements for the same parameters were less pronounced (34\% for stiffness, $44 \%$ for thickness and $50 \%$ for irregularity).

\section{Discussion}

The physiopathology of striae remains unclear and a challenging issue for aesthetic medicine. The etiological mechanisms proposed relate to hormones, and structural alterations to the integument. However, it may involve

Table 3. Parameters of PSAS - mean values and results for the comparison of treatments and measurement time points by Wilcoxon signed-ranks test $(\mathrm{N}=32)$.

\begin{tabular}{|c|c|c|c|c|c|c|}
\hline \multirow{3}{*}{ Parameter of PSAS } & \multirow{3}{*}{ Time } & \multicolumn{2}{|c|}{ Mean values } & \multicolumn{3}{|c|}{ p-values } \\
\hline & & \multirow[b]{2}{*}{ Untreated } & \multirow[b]{2}{*}{ P-3059 } & \multirow[b]{2}{*}{$\begin{array}{c}\text { Between } \\
\text { treatments }\end{array}$} & \multicolumn{2}{|c|}{ Between times } \\
\hline & & & & & Untreated & P-3059 \\
\hline \multirow{2}{*}{ Are the striae painful? ${ }^{* *}$} & Day 1 & $1.0 \pm 0.0$ & $1.0 \pm 0.0$ & -- & \multirow{2}{*}{1.000} & \multirow{2}{*}{1.000} \\
\hline & Day 57 & $1.0 \pm 0.0$ & $1.0 \pm 0.0$ & 1.000 & & \\
\hline \multirow{2}{*}{ Are the striae itching? ${ }^{* *}$} & Day 1 & $1.0 \pm 0.0$ & $1.0 \pm 0.0$ & - & \multirow{2}{*}{1.000} & \multirow{2}{*}{1.000} \\
\hline & Day 57 & $1.0 \pm 0.0$ & $1.0 \pm 0.2$ & 1.000 & & \\
\hline \multirow{2}{*}{$\begin{array}{l}\text { Is the color of the striae different from the } \\
\text { color of your normal skin?*** }\end{array}$} & Day 1 & $5.0 \pm 1.7$ & $5.1 \pm 1.8$ & -- & \multirow{2}{*}{0.980} & \multirow{2}{*}{0.318} \\
\hline & Day 57 & $4.9 \pm 2.0$ & $4.7 \pm 2.0$ & 0.231 & & \\
\hline \multirow{2}{*}{$\begin{array}{l}\text { Is the stiffness of the striae different from } \\
\text { your normal skin? }\end{array}$} & Day 1 & $4.8 \pm 2.0$ & $4.7 \pm 1.9$ & - & \multirow{2}{*}{0.987} & \multirow{2}{*}{0.154} \\
\hline & Day 57 & $4.7 \pm 1.9$ & $4.2 \pm 2.0$ & 0.166 & & \\
\hline \multirow{2}{*}{$\begin{array}{l}\text { Is the thickness of the striae different from } \\
\text { your normal skin? }\end{array}$} & Day 1 & $5.2 \pm 2.0$ & $5.1 \pm 1.9$ & -- & \multirow{2}{*}{0.228} & \multirow{2}{*}{0.044} \\
\hline & Day 57 & $4.8 \pm 2.0$ & $4.5 \pm 1.7$ & 0.386 & & \\
\hline \multirow{2}{*}{$\begin{array}{l}\text { Are the striae more irregular than your } \\
\text { normal skin?** }\end{array}$} & Day 1 & $5.8 \pm 1.9$ & $5.9 \pm 1.9$ & -- & \multirow{2}{*}{0.019} & \multirow{2}{*}{0.056} \\
\hline & Day 57 & $5.0 \pm 1.8$ & $5.1 \pm 2.2$ & 0.856 & & \\
\hline
\end{tabular}

\footnotetext{
${ }^{* *}$ Score: 1 = no, not at all to $10=$ yes, very much; ${ }^{* * *}$ Score: $1=$ no, as normal skin to $10=$ yes, very different.
} 


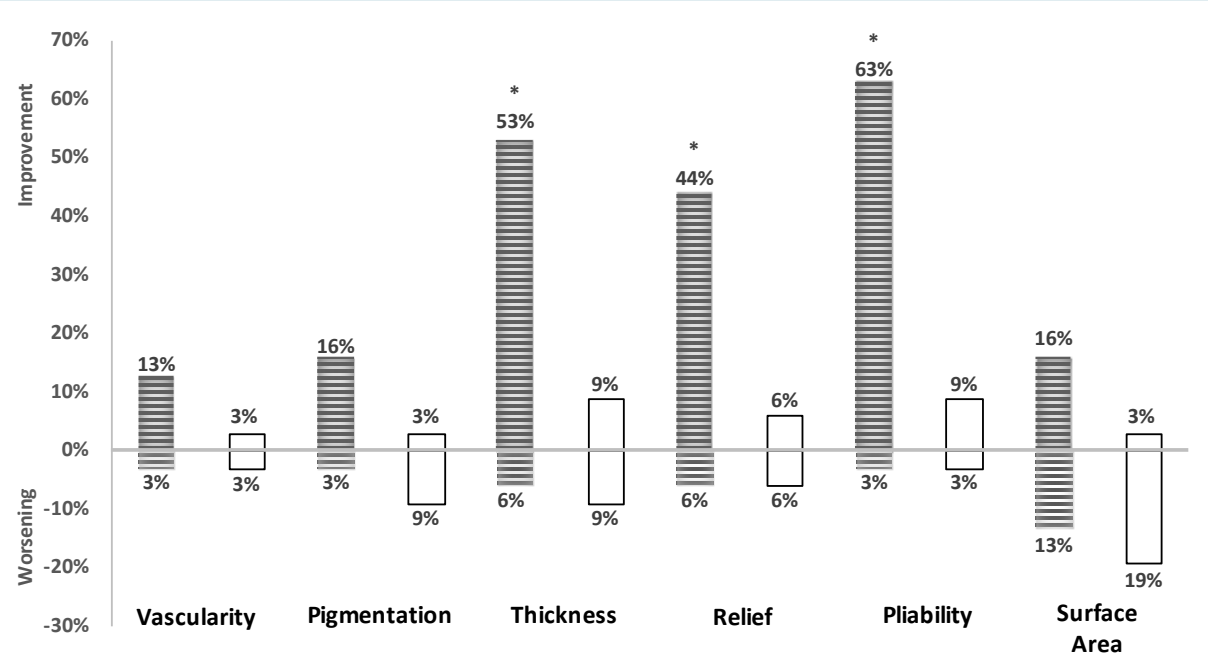

Figure 1. Counts of subjects with improvement and worsening for parameters of OSAS $(\mathrm{N}=$ 32) in P-3059 (shadow histogram) and in the untreated (blank histogram). Asterisks represent significant differences between treatments (Fisher's test).

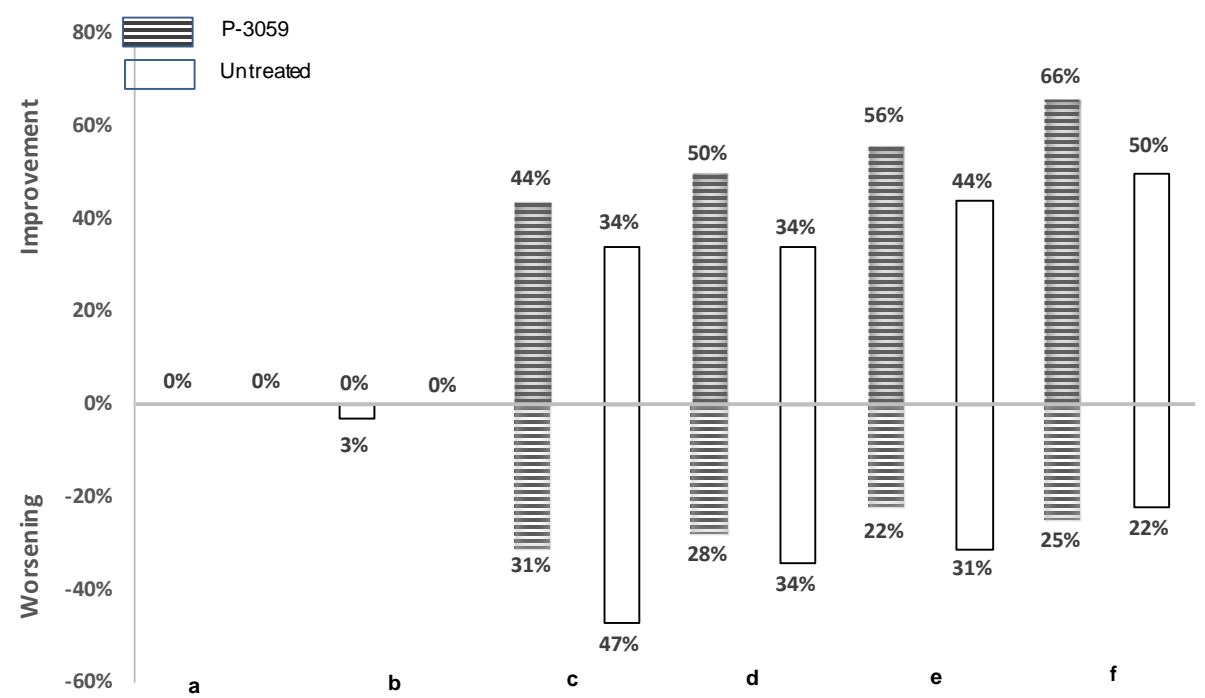

Figure 2. Counts of subjects with improvement and worsening for parameters of PSAS ( $\mathrm{N}=$ 32), where a, b, c, d, e and f refer to pain, itching, color (pigmentation and vascularity), pliability, thickness and relief, respectively.

stretching of the skin, causing lesions in fibrilin microfibrils, which in younger spurt women are likely to be more fragile and are therefore more susceptible to rupture [14]. Nowadays, a number of therapies are available for such conditions: tretinoin, lasers, surgery and many more, are the most common treatment modalities, but they often present some disadvantages related to their efficacy or to their safety profile.

The purpose of this study was to evaluate the aesthetic efficacy of a cosmetic product, P-3059, exploiting its elasticizing and repairing properties, due to its composition based on carboxymethylcysteine, Vitamin $\mathrm{E}$ and sweet almond oil, on reducing striae on the body and legs of female subjects after an 8-week treatment. This was our biggest challenge, as topical treatments are in most of cases indicated in the prevention of striae occurrence instead of in the treatment of full-blown striae, where laser treatment or surgery could be more efficacious within their own limits.

Although the eligibility criteria foresaw women between 18 and 65 years old, nevertheless our population was rather homogeneous with the subjects included between 19 and 45 years old. Moreover, in order to avoid any bias in the study results, due to any rapid weight gain or loss, prior or throughout the course of the study, the 
weight monitoring was guaranteed during the study. Our results showed an improvement in the clinical appearance of striae treated with P-3059 on the three parameters out of six parameters in the OSAS composite endpoint that P-3059 might reasonably be expected to have an effect on. These are the three parameters where P-3059 might be expected to work. There were statistically significant improvements in striae thickness, relief and pliability following treatment with P-3059, both versus baseline and in comparison to the untreated area, as assessed objectively by a Trained Evaluator. In addition, $53 \%$ of subjects had an improvement in striae thickness, $44 \%$ an improvement in striae relief and 63\% an improvement in striae pliability after treatment with P-3059. The self-assessment evaluation, done directly by the subjects, also revealed a statistical superiority of P-3059 over placebo control in the judgement of striae thickness.

Some potential limitations to this study should be mentioned: the study was randomized within subjects, but it followed an open design, which may have influenced the subject self-assessment, resulting in a study bias. However, what may have represented a limitation appeared not to be, as the Evaluator's assessment was even more favorable than the self-assessment. A second limitation may be due to the small sample size. This would explain why in some clinical parameters, besides the positive trend, there were no statistically significant differences. However, some objective parameters achieved statistical significance despite the small sample size, demonstrating the value of the trial as a preliminary study. A longer treatment period may also have improved the statistical findings.

Although POSAS score is a validated tool for striae assessment, some considerations have to be made. The observed difference between the OSAS and the PSAS score is in agreement with some recurrent findings of the literature, which tend to undervalue the influence of the subject's satisfaction, compared to trained evaluator assessment [15]. This is due to the fact that the individuals assessed their striae in a highly subjective, various, and difficult to capture way, resulting in a weak intersubjective comparability of judgments. Many factors contribute to the determination of patient's satisfaction: color, thickness and striae irregularity were the significant contributing parameters to the overall subject satisfaction as they were easier to be evaluated visually, in respect to pain, itching and stiffness, which are parameters linked to the subjects' personal perception.

Despite those limitations, the test product, P-3059, significantly improved striae according to the Observer Scar Assessment Scale. This study opens up new perspectives as carboxymethylcysteine was used to treat striae for the first time, taking advantage of the mechanism of action of P-3059. In fact, the product has the capability to decrease the rigidity of protein structures, by breaking the disulphuric bonds, resulting in an increase in the extensibility of the protein molecule. This result is achieved by acting both on ternary and quaternary structures, without affecting the primary and the secondary ones, and is therefore reversible. It seems that this action is not only efficacious in the very well-known therapy for bronchitis, to decrease sputum viscosity, but it could also be applicable in the cosmetic field to improve skin softness. Notably, unless cosmetic approaches to improved appearance of striae alone, P-3059 had the effect of improving both the appearance and distressing physical characteristics of striae, while the emollients do not.

\section{Conclusion}

In conclusion, the current study represents an attempt to use a product containing carboxymethylcysteine, using a validated assessment scale, to examine overall patient satisfaction in female subjects with striae in their bodies/legs. The results reported herein, showed that in the study population, the treatment with P-3059 produces a statistically significant improvement in Observer Scar Assessment Scale, with particular emphasis on improved appearance and physical characteristics of striae, such as reducing striae thickness, improving relief and pliability of striae, as well as, subject satisfaction linked to a reduction in the thickness of the striae, when compared to an untreated area. If confirmed by further, larger studies, this result could open a new way forward in the treatment of mild to moderate striae.

\section{References}

[1] Yamaguchi, K., Suganuma, N. and Ohashi, K. (2012) Quality of Life Evaluation in Japanese Pregnant Women with Striae Gravidarum: A Cross-Sectional Study. BMC Research Notes, 5, 450. http://dx.doi.org/10.1186/1756-0500-5-450

[2] Salter, S.A. and Kimball, A.B. (2006) Striae Gravidarum. Clinics in Dermatology, 24, 97-100. http://dx.doi.org/10.1016/j.clindermatol.2005.10.008

[3] Brennan, M., Young, G. and Devane, D. (2012) Topical Preparations for Preventing Stretch Marks in Pregnancy. 
Cochrane Database of Systematic Reviews, 11, Article ID: CD000066. http://dx.doi.org/10.1002/14651858.cd000066.pub2

[4] “Stretch Mark” (2009). Encyclopædia Britannica.

[5] Sheu, H.M., Yu, H.S. and Chang, C.H. (1991) Mast Cell Degranulation and Elastolysis in the Early Stage of Striae Distensae. Journal of Cutaneous Pathology, 18, 410-416. http://dx.doi.org/10.1111/j.1600-0560.1991.tb01376.x

[6] Ash, K., Lord, J., Zukowski, M. and McDaniel, D.H. (1998) Comparison of Topical Therapy for Striae Alba (20\% Glycolic acid/0.05\% Tretinoin versus 20\% Glycolic Acid/10\% L-Ascorbic Acid). Dermatologic Surgery, 24, 849-856. http://dx.doi.org/10.1111/j.1524-4725.1998.tb04262.x

[7] Alldredge, B.K., Corelli, R.L., Ernst, M.E., Guglielmo, B.J., Jr., Jacobson, P.A., Kradjan, W.A. and Williams, B.R. (2013) Koda-Kimble and Young's Applied Therapeutics: The Clinical Use of Drugs. 10th Edition, Wolters Kluwer Health/Lippincott Williams \& Wilkins, Baltimore.

[8] Hernandez-Perez, E., Colombo-Charrier, E. and Valencia-Ibiett, E. (2002) Intense Pulsed Light in the Treatment of Striae Distensae. Dermatologic Surgery, 28, 1124-1130.

[9] Alexiades-Armenakas, M.R., Bernstein, L.J., Friedman, P.M. and Geronemus, R.G. (2004) The Safety and Efficacy of the $308 \mathrm{~nm}$ Excimer Laser in Pigment Correction of Hypopigmented Scars and Striae Alba. Archives of Dermatology, 140, 955-960. http://dx.doi.org/10.1001/archderm.140.8.955

[10] Kim, B.J., Lee, D.H., Kim, M.N., Song, K.Y., Cho, W.I., Lee, C.K., et al. (2008) Fractional Photothermolysis for the Treatment of Striae Distensae in Asian Skin. American Journal of Clinical Dermatology, 9, 33-37. http://dx.doi.org/10.2165/00128071-200809010-00003

[11] Alderman, A.K., Collins, E.D., Streu, R., Grotting, J.C., Sulkin, A.L., Neligan, P., et al. (2009) Benchmarking Outcomes in Plastic Surgery: National Complication Rates for Abdominoplasty and Breast Augmentation. Plastic and Reconstructive Surgery, 124, 2127-2133. http://dx.doi.org/10.1097/prs.0b013e3181bf8378

[12] Ud-Din, S., McAnelly, S.L., Bowring, A., et al. (2013) A Double-Blind Controlled Clinical Trial Assessing the Effect of Topical Gels on Striae Distensae (Stretch Marks): A Non-Invasive Imaging, Morphological and Immunohistochemical Study. Archives of Dermatological Research, 305, 603-617. http://dx.doi.org/10.1007/s00403-013-1336-7

[13] Van der Wal, M.B., Tuinebreijer, W.E., Bloemen, M.C., Verhaegen, P.D., Middelkoop, E. and van Zuijlen, P.P. (2012) Rasch Analysis of the Patient and Observer Scar Assessment Scale (POSAS) in Burn Scars. Quality of Life Research, 21, 13-23. http://dx.doi.org/10.1007/s11136-011-9924-5

[14] Valente, D.S., Zanella, R.K., Doncatto, L.F. and Padoin, A.V. (2014) Incidence and Risk Factors of Striae Distensae Following Breast Augmentation Surgery: A Cohort Study. PLoS ONE, 9, e97493. http://dx.doi.org/10.1371/journal.pone.0097493

[15] Consorti, F., Mancuso, R., Piccolo, A., Pretore, E. and Antonaci, A. (2013) Quality of Scar after Total Thyroidectomy: A Single Blinded Randomized Trial Comparing Octyl-Cyanoacrylate and Subcuticular Absorbable Suture. ISRN Surgery, 2013, Article ID: 270953. http://dx.doi.org/10.1155/2013/270953 